\title{
ADVANCED SHAPE FINDING ALGORITHM OF FORCE DENSITY METHOD BASED ON FEM
}

\author{
K.S. Lee ${ }^{1, *}$ and S.E. $\operatorname{Han}^{2}$ \\ ${ }^{1}$ Research Assistant Professor, Department of Architectural Engineering, School of Architecture \\ Inha University, 253 Yonghyundong, Nam-gu, Incheon, 402-751, South Korea \\ ${ }^{2}$ Professor, Department of Architectural Engineering, School of Architecture \\ Inha University, 253 Yonghyundong, Nam-gu, Incheon, 402-751, South Korea \\ *(Corresponding author: E-mail: lks1838@naver.com)
}

Received: 28 October 2010; Revised: 8 March 2011; Accepted: 15 March 2011

\begin{abstract}
This paper proposes a modified formulation of the force density method by adopting finite element method procedures and replacing each edge of a 3-node or 4-node membrane element with a linear line element. The membrane element model, not the cable-net model, is used for simultaneous shape finding and load analysis. The derived force density matrix has a banded, symmetric nature to ensure its effectiveness in an iterative procedure. By using the nonlinear shape finding option, a number of nonlinear shape finding problems can be solved for each force density mode controlling the shape of the surface. Therefore, it is needs not to use the nonlinear numerical method such as NR or DR method. Therefore, the present research may improve the effectiveness and applicability of the FDM in linear and nonlinear shape finding problems. The following numerical examples will verify the various excellent numerical abilities of the proposed FDM.
\end{abstract}

Keywords: Tension structures, Shape finding, Force density method, Equally stressed surface, Geodesic surface

\section{INTRODUCTION}

The force density method (FDM) originally proposed by Linkwitz [1] and Sheck [2], and extended by Grundig and Bahndorf [3] and Grundig et al. [4] to solve the equilibrium for any type of cable-net structure has also been used as a linear shape finding strategy for architectural fabric tension structures conveniently. The advantage of the method is that it provides a linear solution to the equilibrium shape finding problem. The final shape obtained by the FDM is independent of the initial coordinates of the structures, and the controlling element variable is that of force density only.

The analysis process is therefore relatively simple compared with the nonlinear process of methods, such as the Newton-Raphson (NR) method [5-9] or the Dynamic Relaxation (DR) method [10-15] for minimum surface analysis. Therefore, it can easily be used in practical projects as a repeated shape finding process with respect to various types of configurations and can be incorporated into specific purpose analysis software such as EASY membrane(Technet), FORTEN 4000, RhinoMembrane $[16,17]$. The main drawback of the FDM is that the final distribution of stress is difficult to control. Therefore, once a form has been found, a vector or matrix method must be used to analyze its response under a load $[10,11,15]$.

Recently, generalizded FDM based on FEM are proposed by researchers [8,9,16-19]. Maurin and Motro [18] proposed the "The surface stress density method" which uses surface triangular elements with an isotropic stress tensor and leads to an iterative procedure which converges on configurations that satisfy the laws of static equilibrium. And Pauletti and Pimenta [19] proposed the "natural force density method" which uses the concept of natural strains for the finite element analysis of membranes [20,21]. And it is the extension of the FDM for the initial shape finding of cable and membrane structures, which leads to the solution of a system of linear equations. Their 
method preserves the linearity of original FDM, and overcomes the difficulties of irregular triangular finite element meshes. Moreover, it can be applied to the nonlinear iterative procedure for an equally stressed or minimum surface analysis as the uniform, isotropic plane Cauchy stress state. Bletzinger et al. [9] proposed the form finding based on the updated reference strategy using the latest finite element technology. And this theory is installed in the commercial software, Rhinomembrane [16,17]

In this paper, we analyzed the linear and nonlinear shape finding problems by adopting the concept of generalized FDM based on FEM that proposed by researchers which has a number of advantages over the conventional FDM. In our approach, we replaced each edge of a 3-node or 4-node membrane element with a linear line element and uses the membrane surface element model rather than the cable-net model for simultaneous shape finding and load stress analysis. Also, the derived FDM has a banded symmetric storage nature that could be applied to the iterative procedure effectively. We solved a number of nonlinear shape finding problems of the equally stressed surface, boundary cable element length control and geodesic line control analysis by controlling each force density mode of the surface shape. By using the nonlinear formulation of FDM of this paper, it is needs not to use the nonlinear numerical method such as NR or DR method. Therefore, the present research may improve the effectiveness and applicability of the FDM in linear and nonlinear shape finding problems. The following numerical examples will verify the various excellent numerical abilities of the proposed FDM.

\section{GOVERNING EQUATION OF NONLINEAR SHAPE FINDING ANALYSIS}

The basic governing equation for a nonlinear shape finding algorithm is the general total potential energy formulation. We briefly describe the governing equation for nonlinear shape finding algorithm in the following.

The total potential energy of a system is the sum of the internal strain energy and the external energy. The external energy can be rewritten as the product of the external load vector $\mathbf{P}$ and the system displacement vector $\mathbf{d}$. As a result, the total potential energy is given by

$$
\boldsymbol{\Pi}=\mathbf{U}-\mathbf{P}^{\mathrm{T}} \mathbf{d}
$$

and the partial differential equation of Eq. 1 is given by

$$
\frac{\partial \boldsymbol{\Pi}}{\partial \mathbf{d}}=\frac{\partial \mathbf{U}}{\partial \mathbf{d}}-\mathbf{P}
$$

The partial differential term for internal strain in Eq. 2 can be expressed as the internal force vector $\mathbf{F}$, which involves the volume integral of the element stress $\boldsymbol{\sigma}$ and the strain-displacement matrix B as follow

$$
\frac{\partial \mathbf{U}}{\partial \mathbf{d}}=\mathbf{F}=\mathbf{R}^{T} \int_{V} \mathbf{B}^{T} \boldsymbol{\sigma} d V
$$

Where $\mathbf{R}$ is a coordinate transformation matrix. By combining Eqs. 2 and 3, the residual or unbalanced force vector $\mathbf{g}$, can be expressed as follow 


$$
\mathbf{g}=-\frac{\partial \mathbf{\Pi}}{\partial \mathbf{d}}=\mathbf{P}-\mathbf{R}^{T} \int_{V} \mathbf{B}^{T} \boldsymbol{\sigma} d V=\mathbf{P}-\mathbf{F}_{m}
$$

From Eq. 4, the tangent stiffness matrix $\mathbf{K}_{t}$ could be derived from the partial derivative of the residual vector $\mathbf{g}$ with respect to the displacement $\mathbf{d}$ as

$$
\mathbf{K}_{t}=\frac{\partial \mathbf{g}}{\partial \mathbf{d}}=\mathbf{R}^{T} \int_{V} d \mathbf{B}^{T} \boldsymbol{\sigma} d V+\mathbf{R}^{T} \int_{V} \mathbf{B}^{T} d \boldsymbol{\sigma} d V
$$

Because the static equilibrium state of the structure is the state of minimum potential energy, the characteristic equation of static equilibrium can be expressed through Eq. 2 as

$$
\frac{\partial \Pi}{\partial \mathbf{I}}=0
$$

Generally, Eq. 6 cannot be exactly satisfied. Therefore, the static equilibrium state is achieved when the convergence criteria of Eq. 4 being close to zero. In the case of shape finding analysis, we introduce the initial prestress $\boldsymbol{\sigma}_{0}$ to control the final shape of structure with the external load vector set to be zero. Hence, the unbalance load vector is derived from the internal force vector of Eqs. 3 and 4 as follow.

$$
\mathbf{g}=-\int_{V} \mathbf{B}^{T} \boldsymbol{\sigma}_{0} d V
$$

Using the nonlinear iterative procedure, the meshes of initial surface are moved to the balanced unknown final configuration when the unbalanced forces converge to zero.

The equally stressed surface that is frequently used as initial surface shape of a membrane is determined by iterative shape finding analysis. It is determined by allowing the norm of the residual vector in Eq. 7 to converge to nearly zero with the initially introduced prestress, which should be continually fixed at a constant value. Usually, the standard NR method with a matrix equation, or conversely, a DR method with a vector equation, can be used to minimize the unbalance force by an iterative procedure. However, for nonlinear shape finding or stabilizing process problems [13], the DR method is appropriate because of its stable and fast vector equations.

\section{FORMULATION OF FDM}

Originally, the equilibrium equation of FDM in $x$-coordinate is described as follows [2]

$$
\left[\mathbf{C}^{T} \mathbf{Q C}\right] \mathbf{X}=\mathbf{P}
$$

Where $\mathbf{C}$ is a rectangular matrix of the element connectivity, $\mathbf{Q}$ is square diagonal element force density coefficient matrix and $\mathbf{X}$ and $\mathbf{P}$ are the $x$-axis coordinates and the load vector, respectively. According to the free and fixed node, the separated form of Eq. 8 is written in Eq. 9 as follow

$\left[\mathbf{C}_{F}: \mathbf{C}_{X}\right]^{T} \mathbf{Q}\left[\mathbf{C}_{F}: \mathbf{C}_{X}\right]\left\{\mathbf{X}_{F}: \mathbf{X}_{X}\right\}=\mathbf{P}$ 
As a result, the free $\mathrm{x}$-axis coordinate of the system can be obtained with the following equations:

$$
\begin{aligned}
& \mathbf{X}_{F}=\mathbf{D}_{F}^{-1} \mathbf{P}_{F}-\mathbf{D}_{F}^{-1} \mathbf{D}_{X} \mathbf{X}_{X} \\
& \mathbf{D}_{F}=\mathbf{C}_{F}^{T} \mathbf{Q} \mathbf{C}_{F} \\
& \mathbf{D}_{X}=\mathbf{C}_{F}^{T} \mathbf{Q} \mathbf{C}_{X}
\end{aligned}
$$

The equilibrium equations for the other two axes (the $y$ - and the $z$-axis) are obtained by expanding the Eqs. 10-12 of x-axis coordinate. The original FDM process is very simple and robust, but the matrix equation of Eqs. 10-12 has sparse storage, so the original FDM formulation may be ineffective in terms of numerical computing.

The FDM is a popular linear shape finding algorithm that is used to determine the initial curved three-dimensional (3D) shape of fabric membranes and cable-net structures. The thickness of a fabric membrane material is very thin, from 1 to $2 \mathrm{~mm}$; therefore, it does not experience compression and shear resistance stiffness and needs to be stabilized by an initially introduced prestress. The FDM uses a linear line element to represent the membrane surface, and the shape finding and load analysis is carried out with only an element force density that is determined from the element prestress divided by the element length. In addition, the Young's modulus of the material is not considered. Therefore, the FDM may be more appropriate for membrane structures as a shape finding method that represents the membrane material characteristics. However, to verify the element stress level under external loads, a nonlinear analysis must be used to analyze its response under load. This is the main drawback of the FDM. A different analysis model has to be prepared to carry out the shape finding and the load analysis.

To overcome this shortcoming, the membrane element is substituted into each of the 3- or 4-edge line elements, and the elementary force density matrix of the membrane element is sub-assembled from each edge line element, as shown in Figure 1. As a result, model of the shape finding problem can be applicable to the nonlinear load analysis problem.

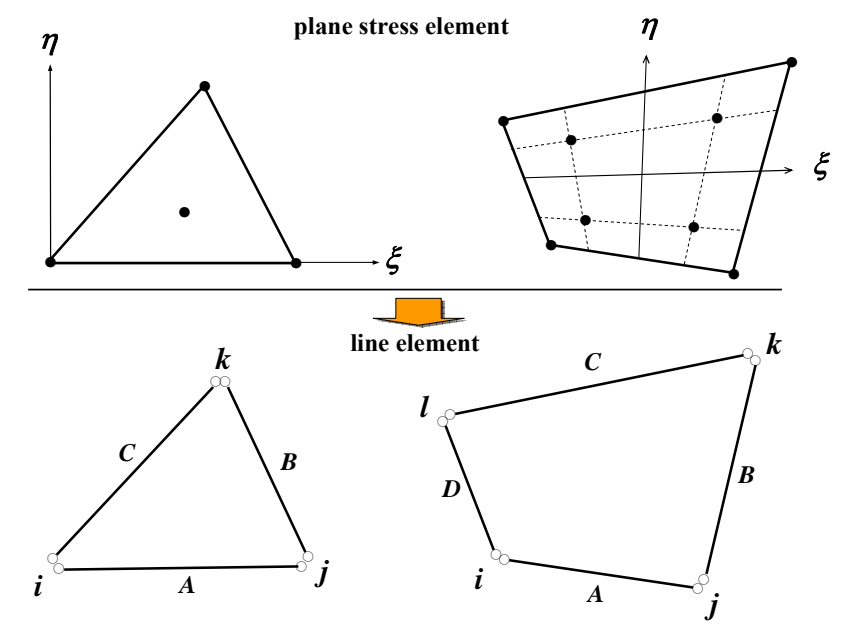

Figure 1. Substitution a Membrane Element for an Edge Line Element 


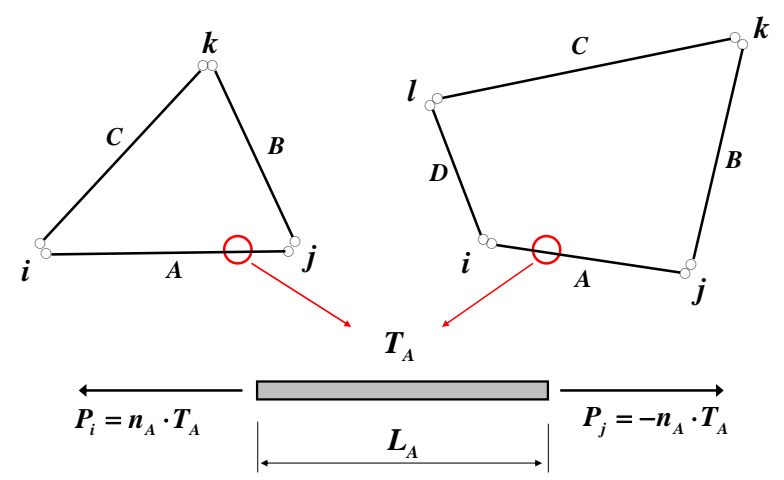

Figure 2. FDM Formulation of a Membrane Line Element

The equilibrium equation of line element- $\mathrm{A}$ in Figure 2 can be written as follow

$$
\begin{aligned}
& P_{i}=n_{A} T_{A}=T_{A}\left(\frac{x_{i}-x_{j}}{L_{A}}\right)=\frac{T_{A}}{L_{A}}\left(x_{i}-x_{j}\right)=q_{A}\left(x_{i}-x_{j}\right) \\
& P_{j}=-n_{A} T_{A}=T_{A}\left(\frac{-x_{i}+x_{j}}{L_{A}}\right)=\frac{T_{A}}{L_{A}}\left(x_{j}-x_{i}\right)=q_{A}\left(x_{j}-x_{i}\right)
\end{aligned}
$$

Where $\mathbf{x}_{i}$ and $\mathbf{x}_{j}$ are the x-axis coordinates at nodes $i$ and $j$, and $n_{A}$ is the direction cosine. In Eq. 13 and 14, the value of $T_{A} / L_{A}$ is defined as the element force density $q_{A}$ of element A which can be described in matrix form as follow

$$
\begin{aligned}
& {\left[\begin{array}{c}
1 \\
-1
\end{array}\right] q_{A}\left[\begin{array}{ll}
1 & -1
\end{array}\right]\left\{\begin{array}{l}
x_{i} \\
x_{j}
\end{array}\right\}=\left\{\begin{array}{l}
P_{i} \\
P_{j}
\end{array}\right\}} \\
& q_{A}\left[\begin{array}{cc}
1 & -1 \\
-1 & 1
\end{array}\right]\left\{\begin{array}{l}
x_{i} \\
x_{j}
\end{array}\right\}=\left\{\begin{array}{l}
P_{i} \\
P_{j}
\end{array}\right\}
\end{aligned}
$$

Eq. 16 can also be written in an alternative form as

$$
\mathbf{D}_{X} \mathbf{X}=\mathbf{P}
$$

$\mathbf{D}_{X}=q_{A}\left[\begin{array}{cc}1 & -1 \\ -1 & 1\end{array}\right]$

Therefore, the extended form of Eq. 17 and 18 for the $\mathrm{x}, \mathrm{y}$ and $\mathrm{z}$-axis can be written as 


$$
\begin{aligned}
& q_{A}\left[\begin{array}{cccccc}
1 & 0 & 0 & -1 & 0 & 0 \\
0 & 1 & 0 & 0 & -1 & 0 \\
0 & 0 & 1 & 0 & 0 & -1 \\
-1 & 0 & 0 & 1 & 0 & 0 \\
0 & -1 & 0 & 0 & 1 & 0 \\
0 & 0 & -1 & 0 & 0 & 1
\end{array}\right]\left\{\begin{array}{c}
x_{i} \\
y_{i} \\
z_{i} \\
x_{j} \\
y_{j} \\
z_{j}
\end{array}\right\}=\left\{\begin{array}{c}
P_{i x} \\
P_{i y} \\
P_{i z} \\
P_{j x} \\
P_{j y} \\
P_{j z}
\end{array}\right\} \\
& \mathbf{D}=\left[\begin{array}{cccccc}
1 & 0 & 0 & -1 & 0 & 0 \\
0 & 1 & 0 & 0 & -1 & 0 \\
0 & 0 & 1 & 0 & 0 & -1 \\
-1 & 0 & 0 & 1 & 0 & 0 \\
0 & -1 & 0 & 0 & 1 & 0 \\
0 & 0 & -1 & 0 & 0 & 1
\end{array}\right]
\end{aligned}
$$

As a result, Eq. 16 and Eq. 19 are the same form of Eq. 8. Therefore, we can obtain the force density matrix of a 3-node or 4-node membrane element of x-axis coordinate by assembling the element of each edge line element of Eq. 18 as

$$
\begin{aligned}
& {\left[\begin{array}{ccc}
q_{A}+q_{C} & -q_{A} & -q_{C} \\
& q_{A}+q_{B} & -q_{B} \\
\text { sym. } & & q_{B}+q_{C}
\end{array}\right]\left\{\begin{array}{l}
x_{i} \\
x_{j} \\
x_{k}
\end{array}\right\}=\left\{\begin{array}{l}
P_{i} \\
P_{j} \\
P_{k}
\end{array}\right\}} \\
& {\left[\begin{array}{cccc}
q_{A}+q_{D} & -q_{A} & 0 & -q_{D} \\
& q_{A}+q_{B} & -q_{B} & 0
\end{array}\right]\left\{\begin{array}{l}
x_{i} \\
x_{j} \\
x_{k} \\
x_{l}
\end{array}\right\}=\left\{\begin{array}{l}
P_{i} \\
P_{j} \\
P_{k} \\
P_{l}
\end{array}\right\}}
\end{aligned}
$$

We can obtain the equilibrium equation for the total system by assembling the element force density matrices of Eq. 16, 21 and 22 for separated free and fixed nodes as

$$
\left[\begin{array}{ll}
\mathbf{D}_{F F} & \mathbf{D}_{F X} \\
\mathbf{D}_{X F} & \mathbf{D}_{X X}
\end{array}\right]\left\{\begin{array}{l}
\mathbf{X}_{F} \\
\mathbf{X}_{X}
\end{array}\right\}=\left\{\begin{array}{l}
\mathbf{P}_{F} \\
\mathbf{P}_{X}
\end{array}\right\}
$$

For free nodes,

$$
\mathbf{D}_{F F} \mathbf{X}_{F}+\mathbf{D}_{F X} \mathbf{X}_{X}=\mathbf{P}_{F}
$$

Therefore, the x-axis coordinate of the shape finding can be obtained from Eq. 24 as

$$
\mathbf{X}_{F}=\mathbf{D}_{F F}^{-1}\left(\mathbf{P}_{F}-\mathbf{D}_{F X} \mathbf{X}_{X}\right)
$$


Eq. 25 can be extended for $y$ - and $z$-axis coordinate systems as well. As a result, the derived $\mathbf{D}_{F F}$ and $\mathbf{D}_{F X}$ in Eqs. 23 and 24 are exactly identical to the $\mathbf{D}_{F}$ and $\mathbf{D}_{X}$ in Eq. 11 and Eq. 12 . The formulation of Eqs. 15-25 which are similar to the FEM, reduce the computational effort of FDM of Eqs. 11 and 12 to model large number of degrees of freedoms. Moreover, the matrix of $\mathbf{D}_{F F}$ in Eq. 25 can be stored in-core or out-of-core in a banded symmetric matrix, not in a sparse matrix, which could increase the computation efficiency remarkably. That formulation based on FEM enables us to use the FDM for the nonlinear shape finding problems.

\section{FDM FORMULATION BASED ON FEM}

In this section, another form of FDM formulation based on FEM is (are)described. This process is based on the well known geometrically nonlinear equation of cable and truss element without material elastic stiffness.

In section 3, we do not consider the material properties of Young's modulus but force density of element only. This approach is known as the generalized FDM formulation based on FEM described in literatures [8,9,16-19].

The tangent stiffness matrix of a cable element is composed by the elastic and geometric stiffness matrices.

But if we consider the geometric stiffness of cable element with isotropic condition of material for ideal case of FDM, the strain of cable element can be written as follow

$$
\varepsilon_{x}^{N}=\frac{1}{2}\left\{\left(\frac{\partial u}{\partial x}\right)^{2}+\left(\frac{\partial v}{\partial x}\right)^{2}+\left(\frac{\partial w}{\partial x}\right)^{2}\right\}=\frac{1}{2}\left\{\left(\frac{u_{j}-u_{i}}{L}\right)^{2}+\left(\frac{v_{j}-v_{i}}{L}\right)^{2}+\left(\frac{w_{j}-w_{i}}{L}\right)^{2}\right\}
$$

From Eq. 26, the geometric stiffness matrix given in Eq. (5) for a cable element in the local coordinate system is derived as

$$
\begin{gathered}
\int_{V} d \mathbf{B}^{T} \sigma d V=\frac{1}{L^{2}}\left(\frac{T_{N}}{A}\right) A L\left[\begin{array}{cccccc}
1 & 0 & 0 & -1 & 0 & 0 \\
0 & 1 & 0 & 0 & -1 & 0 \\
0 & 0 & 1 & 0 & 0 & -1 \\
-1 & 0 & 0 & 1 & 0 & 0 \\
0 & -1 & 0 & 0 & 1 & 0 \\
0 & 0 & -1 & 0 & 0 & 1
\end{array}\right]\left\{\begin{array}{c}
u_{i} \\
v_{i} \\
w_{i} \\
u_{j} \\
v_{j} \\
w_{j}
\end{array}\right\} \\
\mathbf{k}_{F}=q_{N}\left[\begin{array}{cccccc}
1 & 0 & 0 & -1 & 0 & 0 \\
0 & 1 & 0 & 0 & -1 & 0 \\
0 & 0 & 1 & 0 & 0 & -1 \\
-1 & 0 & 0 & 1 & 0 & 0 \\
0 & -1 & 0 & 0 & 1 & 0 \\
0 & 0 & -1 & 0 & 0 & 1
\end{array}\right]
\end{gathered}
$$


The geometric stiffness matrix of the global coordinate system can be written as follow

$$
\begin{aligned}
& \mathbf{K}_{F}=\mathbf{R}^{T} \mathbf{K}_{F} \mathbf{R} \\
& =\left[\begin{array}{cc}
\mathbf{K}_{F}^{0} & -\mathbf{K}_{F}^{0} \\
-\mathbf{K}_{F}^{0} & \mathbf{K}_{F}^{0}
\end{array}\right] \\
& \mathbf{K}_{F}^{0}=q_{N}\left[\begin{array}{lll}
1 & 0 & 0 \\
0 & 1 & 0 \\
0 & 0 & 1
\end{array}\right]
\end{aligned}
$$

The local and global geometric stiffness of Eq. 28 and Eq. 29 are exactly identical value, because the local geometric stiffness matrix is composed of diagonal term only. Therefore, the coordinate transformation matrix $\mathbf{R}$ does not need to be considered. The derived geometric stiffness matrix in Eq. 30 is exactly the same as the force density matrix from Eq. 20, so we can independently reduce the geometric stiffness matrix or force density matrix $\mathbf{K}_{F}$ of each elements into $\mathrm{x}, \mathrm{y}$ and z-axis components as shown in Eq. 18 in the FDM formulation for a cable element.

As a result, we can reduce the size of system matrix and the bandwidth of the FDM that was introduced in previous section. It is not necessary to use the full size of the matrix in Eq. 20 or 30, which is the advantage of the FEM-based FDM formulation in this section.

The nonlinear equilibrium equation shown in Eq. 4 can also be used to obtain the incremental displacement $\delta \mathbf{d}$ of the $x$-axis as

$$
\mathbf{P}-\mathbf{R}^{T} \int_{V} \mathbf{B}^{T} \sigma d V=\mathbf{K}_{F} \delta \mathbf{d}
$$

Where $\mathbf{K}_{F}$ is the assembled, reduced force density matrix and the deformed configurations (or in other words, the shape finding results) are simply

$$
\mathbf{X}=\mathbf{X}_{0}+\delta \mathbf{d}
$$

Eqs. 31 and 32 are repeated for the y and z-axis coordinate systems.

The shape (or coordinates) of the membrane structures that is obtained from Eq. 32 is the same as that obtained from Eq. 10 and Eq. 25 for a linear or nonlinear shape finding analysis. Alternatively, we can obtain the same result from the axis-separated Eq. 31 for the $\mathrm{x}, \mathrm{y}$ and $\mathrm{z}$-axis coordinates by using the full matrix of the FEM formulation rather than the reduced matrix, although with less computational efficiency.

From the above equations, we have shown that the shape finding results from the FDM can be obtained from the well-known FEM routines for geometrically nonlinear equations describing a cable element. Therefore, it is a very interesting that the FDM can be understood as a particular formulation of a cable finite element. Moreover, anyone could analyze the shape finding of membrane cable-net structures with only minor changes or even without changing their own FEM code which it used reduced, banded, symmetric matrix operations. 


\section{LINEAR AND NONLINEAR SHAPE FINDING ANALYSIS ACCORDING TO THE FORCE DENSITY MODE}

In FDM theory, the force density is a unique parameter to control the resulting shape of surface, the equilibrium equation and the resulting shape may be affected by the applied force density mode of the FDM. The various formulation of force density modes are proposed and explained in papers $[18,19]$ and commercial software (Forten 4000, Easy, Rhinomembrane [16,17]) by their unique methodologies. The relationship between force density and equilibrium equation of system may be linear and nonlinear with respect to the element length. Therefore, various methods of shape finding approach can be developed. In this section, we describe our various linear and nonlinear shape finding techniques of FDM based on previous researches.

\subsection{Shape Finding Analysis with Constant Force}

\subsubsection{Linear shape finding analysis with constant force mode}

The conventional force density $q$ is determined the unit prestress force of element $T$ divided by element length $L$ as follow

$q=\frac{T}{L}$

The force density of Eq. 33 may be a nonlinear equation for a structural coordinate system because the element length is used to calculate the force density. However, if we pre-define the force density of Eq. 33 as fixed value, the formulation of FDM is linear equation in Eqs. 10, 25 and 31. The identical result of shape finding can be obtained by using different equations of FDM in Eqs. 10, 25 and 31. The linear equation of FDM is originally proposed by Linkwitz [1], Sheck [2] and Grundig and Bahndorf [3] and Grundig et al. [4].

\subsubsection{Nonlinear shape finding analysis: geodesic shape finding}

Originally, the FDM is not linear equation of equilibrium but nonlinear equation by using the Eq. 33.

If we now apply the actual nonlinear equation of Eq. 33 with actual element length $L$ and constant force $T$, then the equilibrium equation of Eq. 25 and Eq. 31 are nonlinear. To solve the nonlinear equation of Eq. 25 and Eq. 31, the iterative numerical processes are required to obtain the equilibrium state. And the resulting equilibrium state of the equation usually leads to a geodesic surface which usually can be obtained by using iterative nonlinear numerical method [10-15]. As mentioned previously, the force density formulation of Eq. 25 and the FDM formulation based on FEM of Eq. 31 show exactly the identical results for nonlinear problems as for linear cases.

\subsection{Shape Finding Analysis with Constant Force Density}

\subsubsection{Linear shape finding analysis: equally stressed surface}

In this case, if we apply the constant element force of Eq. 34 for the force density of element, then the Eqs. 10, 25 and 31 yields actually linear equilibrium equations that need not to iterate to solve the equation. The force density is independent of the structural geometry as a result.

$q=T$ 
We can obtain the theoretical equally stressed surface with constantly introduced prestress for all membrane or cable-net elements by using linear analysis with the force density mode of Eq. 34 .

\subsubsection{Nonlinear shape finding analysis: cable length control}

Because the force density in Eq. 34 is independent of the structural coordinates and the element length, it is possible to analyze the boundary element length control process by using nonlinear equilibrium equation of Eq. 25 or 31 with the force density mode in Eq. 34. The iterative updated element force converges when the designated element length is satisfied.

$$
\begin{aligned}
& \frac{T_{i}-T_{i-1}}{T_{i-1}}=\alpha\left(\frac{L_{D}-L_{i-1}}{L_{i-1}}\right) \\
& T_{i}=T_{i-1}+\alpha T_{i-1}\left(\frac{L_{D}-L_{i-1}}{L_{i-1}}\right)
\end{aligned}
$$

Where $L_{D}$ is the destination element length and $\alpha$ is the scale factor for controlling the convergence rate; a value of 5.0 was used in this study. The boundary cable element length control is implemented in commercial software, Forten 4000.

\section{NUMERICAL EXAMPLES}

The shape finding process is a unique feature of the tension membrane structure that is used as a basic step for load stress analysis and pattern generation. The main objective of shape finding is obtaining the 3D curved surface of structures. Therefore, there is no need to address the complex, nonlinear behavior of the fabric material. Rather, the proper geometric behavior should be considered. In a sense, the FDM is a meaningful algorithm that fulfills such a need. It is a very simple and effective method for shape finding. The numerical algorithm described in this paper will increase the capacity and applicability of the FDM in various ways. To demonstrate the potential efficiency and applicability of this study, a number of results for numerical examples are tested.

\subsection{Numerical Characteristics of the Proposed Algorithm}

Figure 3 illustrates the shape finding result of this example that is composed of a cable-net element. Table 1 shows the numerical characteristics of total DOF, the bandwidth, the size of the system matrix and the number of linear equations for various FDM algorithms that produce the exact identical shape finding result.

In the case of the original FDM, a sparse matrix equation such as the conjugated gradient method needs to be considered in order to handle the sparse nature of the system matrix equations [3]. However, by using the equations of Eq. (25) or Eq. (31) of $\mathbf{D}_{F F}$ and $\mathbf{D}_{F}$. The equations of system matrix become reduced band symmetric matrix by assembling the element force density matrix for each coordinate axis. The total DOF and bandwidth may be reduced by one-third compared with the FEM. It provides effective and fast computational storage and run-time.

In the case of FEM, the analysis may be less effective than using the modified FDM (Eq. 25) or the FDM based on FEM (Eq. 31). However, we can utilize well-defined conventional FEM routines without change or modification. Also, there are many in-core and out-of-core band solver libraries that can increase the efficiency. 
Table 1. Comparison of the Numerical Characteristics for Each FDM Algorithm

\begin{tabular}{|c|c|c|c|c|}
\hline \multirow{2}{*}{$\begin{array}{c}\text { Original } \\
\text { FDM } \\
\text { Modified } \\
\text { FDM }\end{array}$} & $1655 * 3=4965$ & - & $1655^{*} 1655$ & 3 times \\
\cline { 2 - 5 } & $1655 * 3=4965$ & 152 & $1655^{*} 152$ & 3 times \\
\hline FDM_FEM & $1655 * 3=4965$ & 152 & $1655^{*} 152$ & 3 times \\
\hline FEM & $1655 * 3=4965$ & $152 * 3=456$ & $4965 * 456$ & 1 time \\
\hline
\end{tabular}

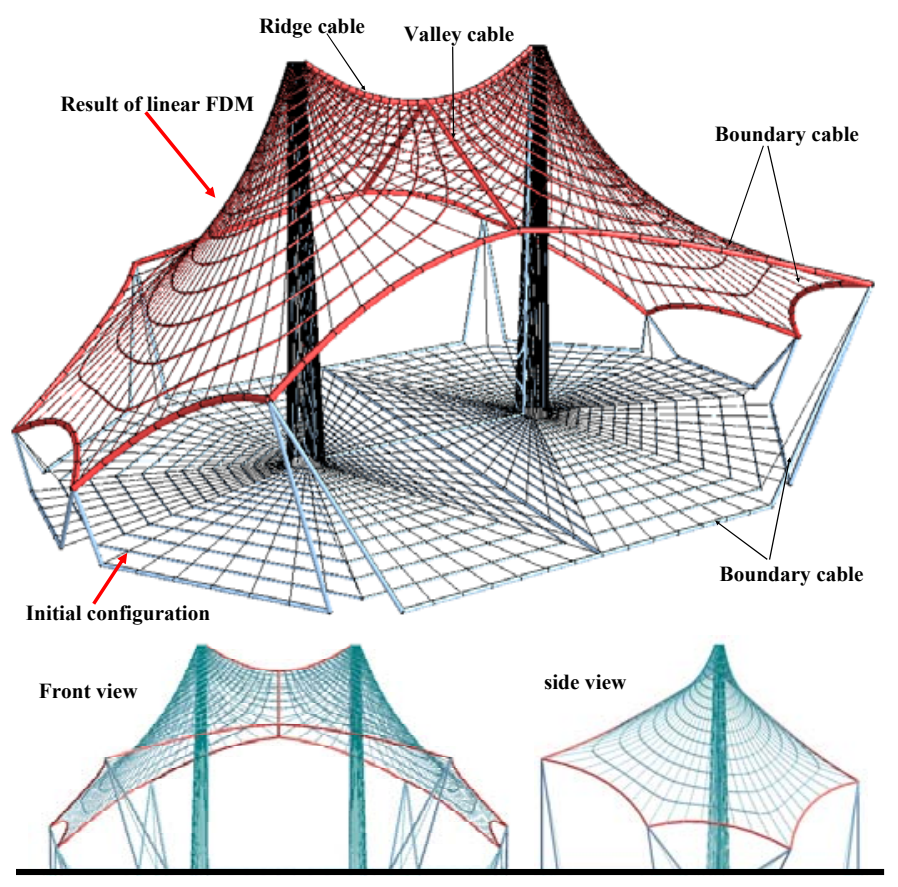

Figure 3. Verification of the Cable-Net Model for Numerical Capacity

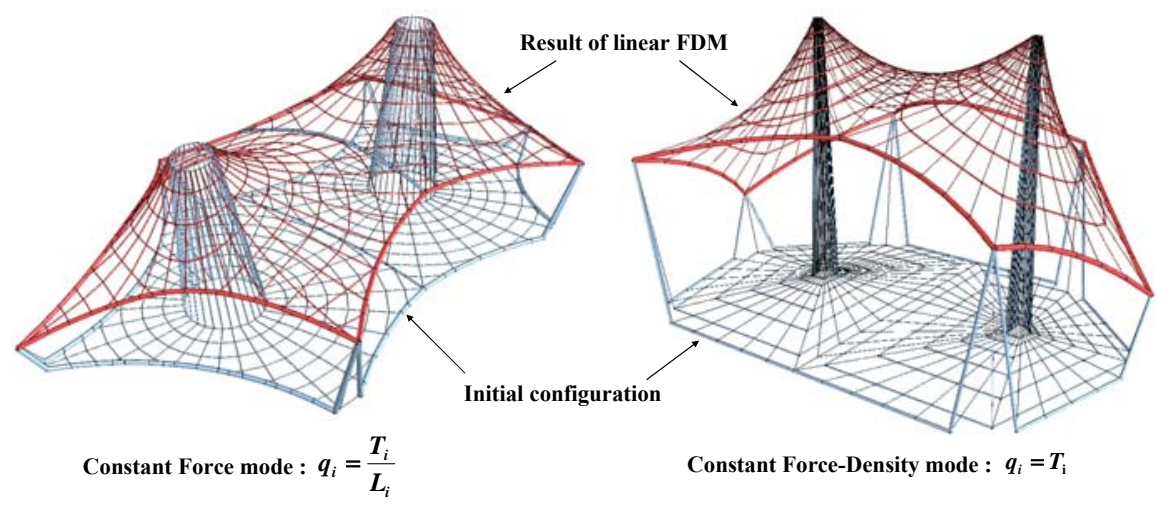

Figure 4. Shape Finding Results According to the Force Density Mode 
Figure 4 shows the results of a linear shape finding analysis according to the force density mode of Eqs. 33 and 34. By using the Eq. 33 with constant force $T_{i}$ and variable element length $L_{i}$, then the equation of force density Eq. 33 is originally nonlinear. However in the FDM formulation, the nonlinear equation of Eq. 33 is assumed to be linear with pre-defined fixed value. The actual linear equation is prevailed when the actual constant force density of Eq. 34 is used. The element length is removed in Eq. 34. Therefore the equation of FDM is actually independent from the geometry of surface and the equation lead to be linear. The resulting shapes of linear equation of FDM with the different force density mode Eq. 33 and Eq. 34 are some different. The shape of Eq. 34 is equally stressed surface of constant force density without the influence of geometry of element length. The shape of Eq. 33 is influenced by the effect of dividing element length. The proper force density mode may be dependent on the initial modeling methodology or the structural geometry. The optimal shape of tension membrane structure is not deterministic but the choice of many possibilities.

\subsection{Shape Finding Analysis of a Saddle-type Surface}

In this section, we explain the linear and nonlinear nature of the force density mode in Eqs. 33 and 34 by carrying out a saddle-type shape finding examples. As explained previously in section 5, the force density mode of Eqs. 33 and 34 may result in linear and nonlinear nature of equilibrium respectively [3].

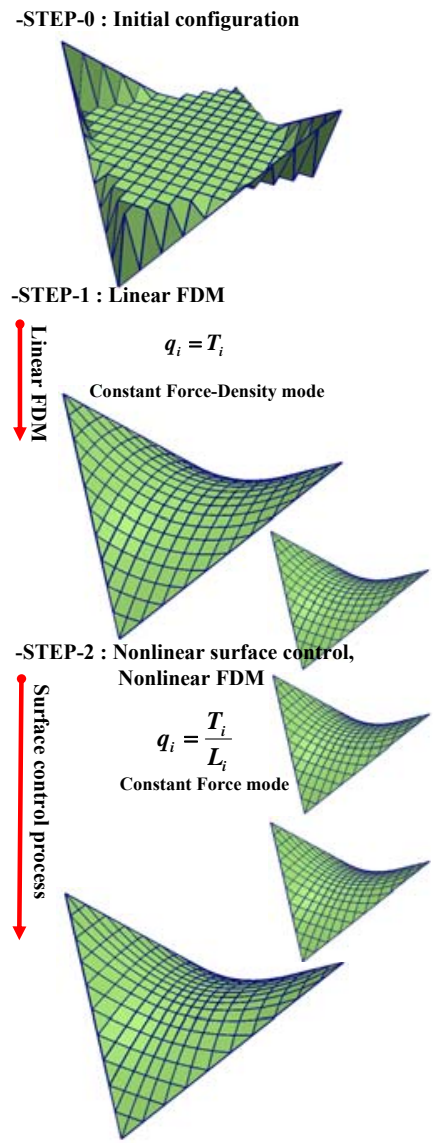

Figure 5. Shape Finding Process for Saddle-1

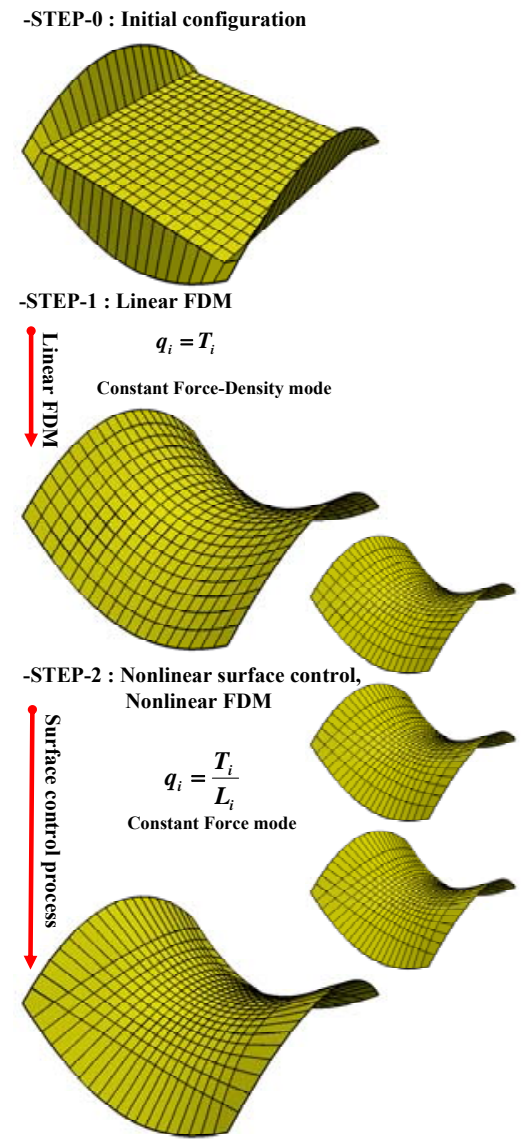

Figure 6. Shape Finding Process for Saddle-2 
The system equation may be a linear when the force density mode of Eq. 34 is adopted. The shape finding analysis with equal prestress to the overall surface elements results in an equally stressed or minimum surface. In the contrast, the system equation may be nonlinear when the force density mode of Eq. 33 is adopted. The iterative analysis with the equation of Eq. 25 or 31 is needed with a reduced band symmetric matrix. The final shape may result in the geodesic surface that has been obtained by using DR geodesic shape finding analyses [10,11]. The geodesic string element of Barnes [10] and Wakefield [11] and the force density mode of Eq. (33) have conceptually and theoretically identical meanings in the nonlinear shape finding process. However the computational effort of FDM in this paper for nonlinear shape finding is more effective than NR or DRM.

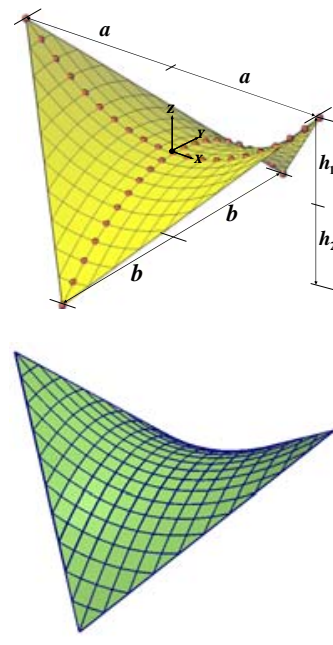

-Linear FDM : Constant Force-Density Mode

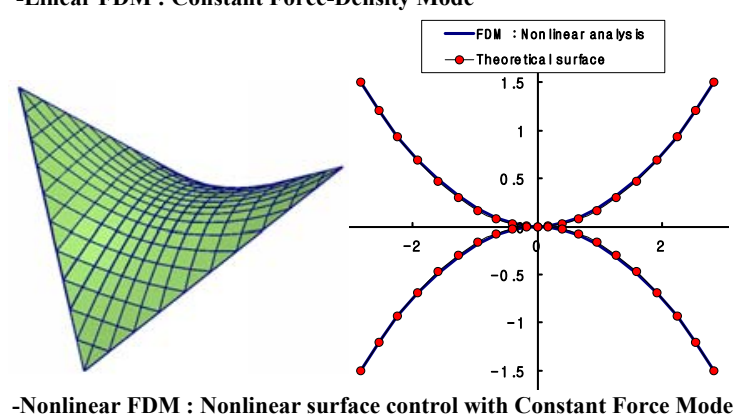
$h_{2}$

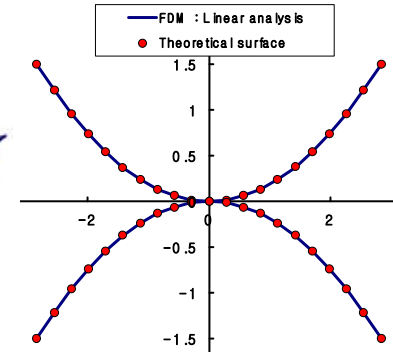

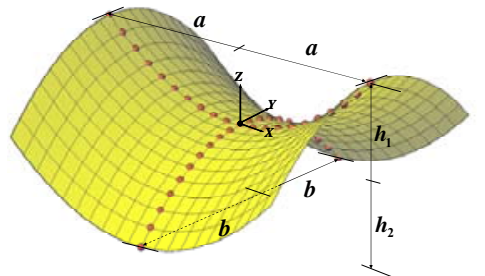
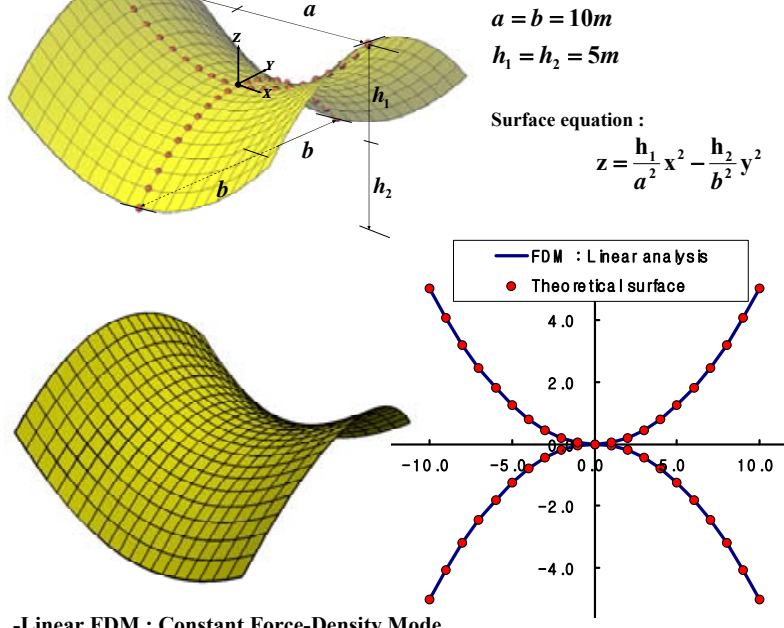

-Linear FDM : Constant Force-Density Mode

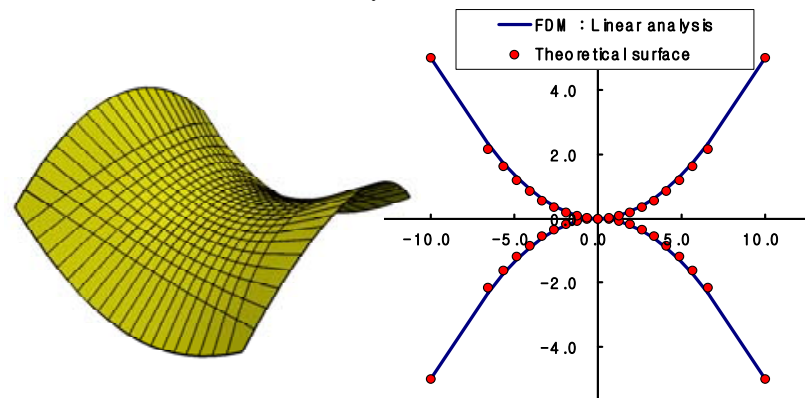

-Nonlinear FDM : Nonlinear surface control with Constant Force Mode

Figure 7. Linear and Nonlinear Shape Finding Result for Saddle-1 and Saddle-2

Two types of saddle surfaces are analyzed, and the results are compared with the theoretical equation given in Eq. 37. The theoretical equation for a saddle-type surface is as follows

$z=\frac{h_{1} x^{2}}{a^{2}}-\frac{h_{2} y^{2}}{b^{2}}$

Figure 5, Figure 6 and Figure 7 show the shape finding process and comparisons with the theoretical values for saddle-1 and saddle-2, respectively. In STEP-1, the linear shape finding analysis is carried out by using the force density mode of Eq. 34. Then the nonlinear process is performed by using Eq. 33. The geodesic surfaces in Figure 5 and Figure 6 are obtained by using proposed nonlinear FDM algorithm. The linear and nonlinear shape finding result as shown in Figure 7 are good agreement with the theoretical value given in Eq. 37. 


\subsection{Practical Application of FDM}

The proposed FDM is applied to a number of practical projects. The equivalent membrane element of Figure 1 is used in the shape finding analysis, and the finite element formulations [5-7] are used in the stress analysis.

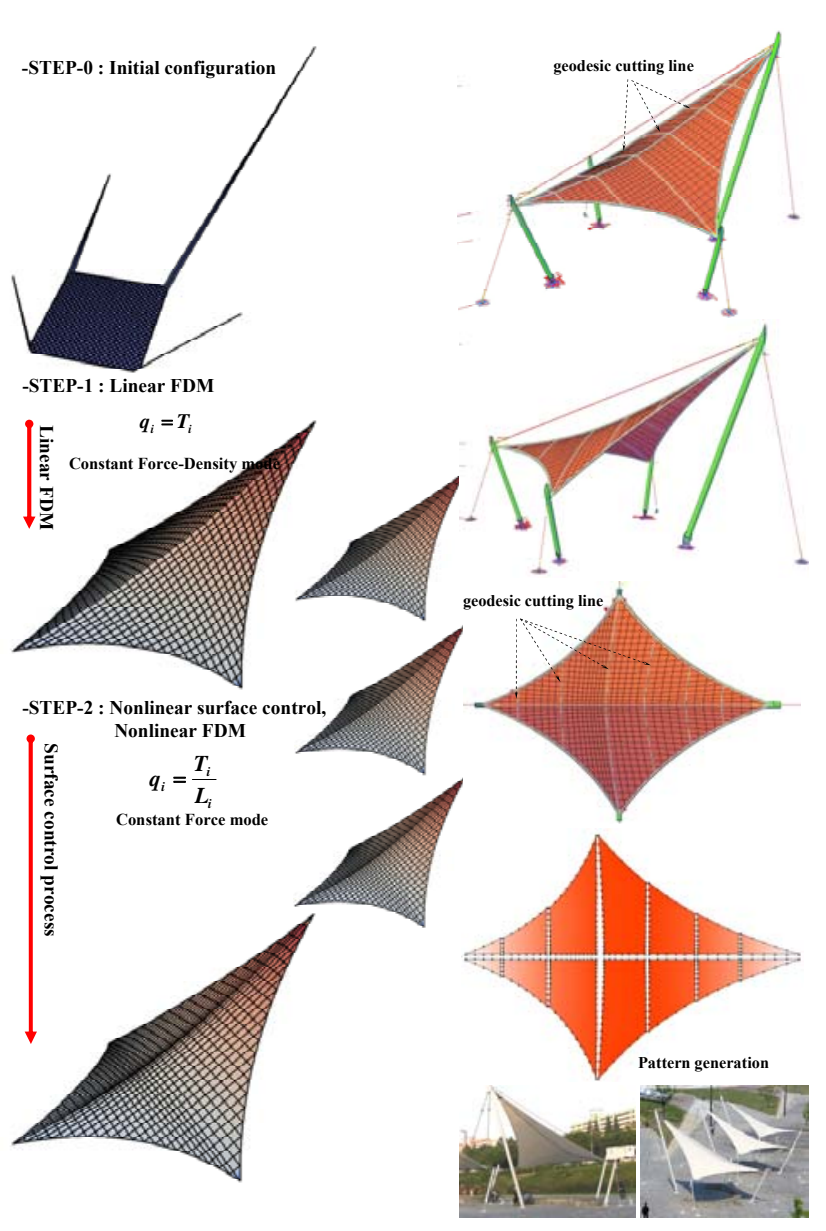

(a) Surface Control

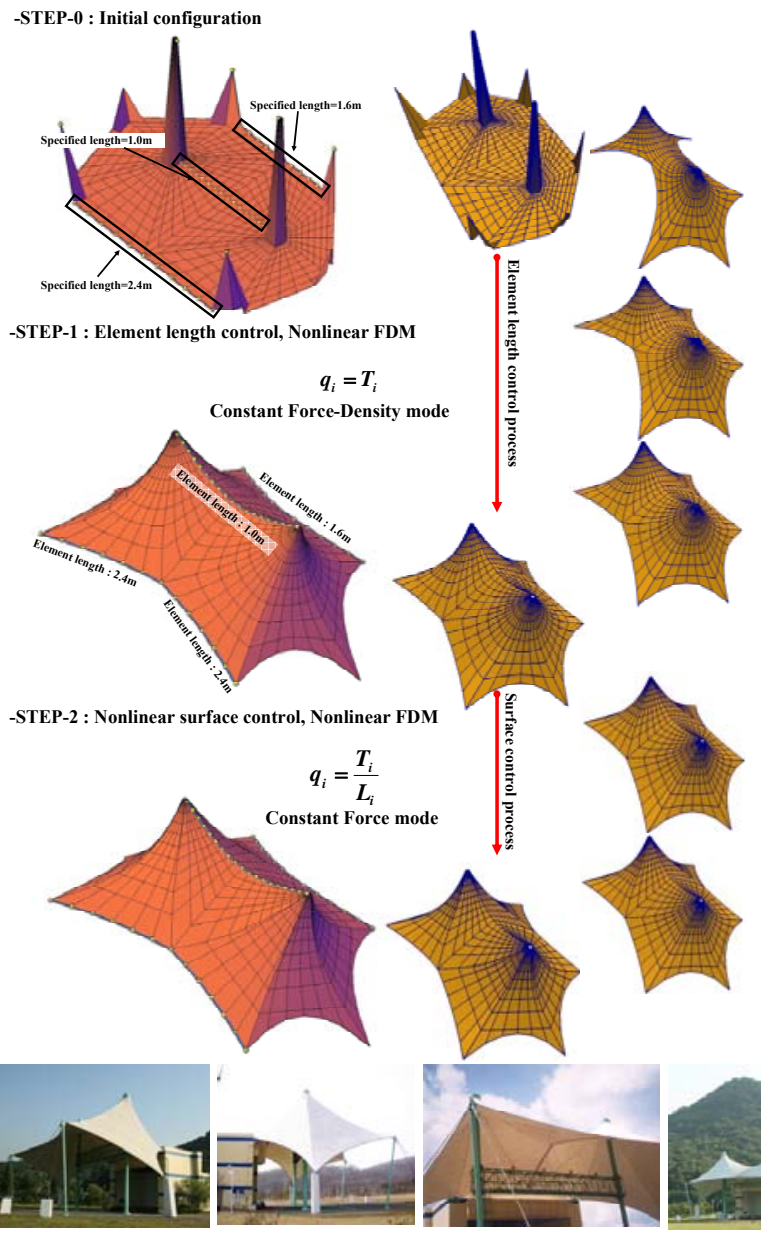

(b) Element Length and Surface Control

Figure 8. Shape Finding Examples of Shelters

Figure 8 shows the shape finding process and an image of a simple shade shelter. The identical process and algorithm of previous examples are applied to the current examples. Also, the geodesic surface obtained from the nonlinear process was used in pattern generation. Figure 8 (b) shows the element length control process of the boundary or ridge cable in a typical 2-pole shelter structure. The prescribed element length $L_{D}$ is introduced in STEP-0 for boundary element length control process of STEP-1 by carrying out the iterative nonlinear process of FDM in Eqs. 35 and 36. The resulting element lengths are listed in Table 2 and show good agreement with the prescribed element length. To perform the element length control process, the linearly independent force density mode with respect to the element length or coordinate should be selected and the initial unit value of prestress is introduced for all cable elements. The final prestress satisfying the prescribed element length is determined by the simple interpolation equation of Eq. 36. Finally, we can carry out the nonlinear geodesic shape finding analysis with the fixed support condition and boundary cable element. Moreover, these algorithms could be applied to large scale roof structures of Figure 
9. The shape finding result is used for the roof geometry, and the following stress analysis and member design process are carried out as shown in Figure 9.
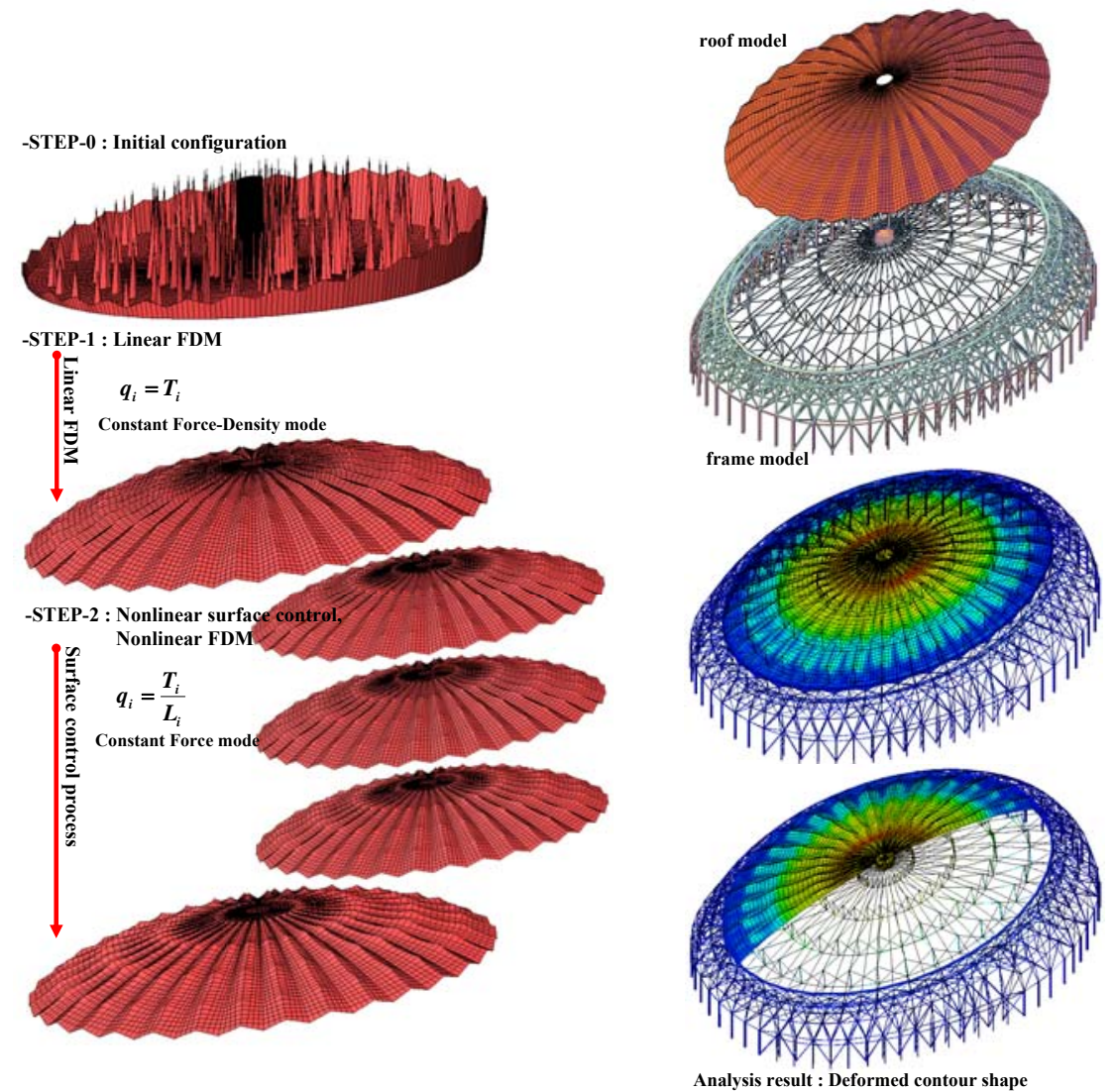

Figure 9. Shape Finding and Load Analysis of Large Scale cable Dome Model

Table 2. Result of Element Length Control Process

\begin{tabular}{|c|c|c|}
\hline ele.No. & $\begin{array}{c}\text { Specified. } \\
\text { Len.(cm) }\end{array}$ & $\begin{array}{c}\text { Result. } \\
\text { Len.(cm) }\end{array}$ \\
\hline 6 & 100.00 & $\mathbf{1 0 0 . 0 0}$ \\
\hline 55 & 160.00 & $\mathbf{1 6 0 . 0 0}$ \\
\cline { 2 - 3 } 73 & 240.00 & $\mathbf{2 4 0 . 0 0}$ \\
96 & 100.00 & $\mathbf{1 0 0 . 0 0}$ \\
\cline { 2 - 2 } 145 & 160.00 & $\mathbf{1 6 0 . 0 0}$ \\
\cline { 2 - 3 } 163 & 240.00 & $\mathbf{2 4 0 . 0 0}$ \\
\hline
\end{tabular}

\section{CONCLUSION}

In this paper, FDM formulation based on FEM are presented and verified for linear and nonlinear shape finding problems. The shape finding process is a unique feature of architectural tension membrane structures that is used as a basic step for load stress analysis and pattern generation. Therefore, the shape finding algorithm should be simple and easy to control the surface geometries. The presented FDM formulation uses the reduced band symmetric matrix assembled from each element force density matrix for simultaneous linear equations. And a well-defined FEM routine could be used for the FDM with minor changes. The concepts of the force density mode and the equivalent membrane element make it possible to conduct a linear or nonlinear analysis of an 
equally stressed surface, a geodesic surface analysis and an element length control process. The resulting surfaces can be successfully used for stress analysis or pattern generation. Therefore, the simple and effective FDM formulation presented in this paper will increase the capacity and applicability of FDM for design and analysis of membrane structures.

\section{ACKNOWLEDGEMENTS}

This research was supported by a grant (code\#'09 R\&D A01) from Cutting-edge Urban Development Program funded by the Ministry of Land, Transport and Maritime Affairs of Korean government.

\section{REFERENCES}

[1] Linkwitz, K., “About Formfinding of Double-curved Structures”, Engineering Structures, 1999, Vol. 21, pp. 709-718.

[2] Sheck, H.J., "The Force Density Method for Form Finding and Computation of General Networks”, Comput. Methods Appl. Mech. Engrg., 1974, Vol. 3, pp. 704-713.

[3] Grundig, L. and Bahndorf, J., "The Design of Wide-span Roof Structures using Micro-computers”, Computers \& Structures, 1988, Vol. 30, No. 3, pp. 495-501.

[4] Grundig, L., Ekert, L. and Moncrieff, E., "Geodesic and Semi-geodesic Line Algorithms for Cutting Pattern Generation of Architectural Texile Structures”, Proc. Asia-Pacific Conf. on Shell and Spatial Structures, Beijing, China, 1996, pp. 435-443.

[5] Ishii, K., "State of the Art Report on Form Finding Problem of Membrane Structures", Research Report on Membrane Structures '89, The Membrane Structures Association of Japan, 1989, Vol. 3, pp. 83-108.

[6] Ishii, K., "State of the Art Report on the Stress Deformation Analysis of Membrane Structures", Research Report on Membrane Structures '90, The Membrane Structures Association of Japan, 1990, Vol. 4, pp. 69-105.

[7] Levy, R. and Spillers, W.R., Analysis of Geometrically Nonlinear Structures, Second Edition, Kluwer Academic Publishers, 2003.

[8] Bletzinger, K.U. and Ramm, E., “A General Finite Element Approach to the Form Finding of Tensile Structures”, Int. Journal of Space Structures, 1999, Vol. 14, No. 2, pp. 131-145.

[9] Bletzinger, K.U., Wuechner, R., Daoud, F. and Camprubi, N., “Computational Methods for Form Finding and Optimization of Shells and Membranes”, Comput. Methods Appl. Mech. Engrg., 2005, Vol. 194, pp. 143-166.

[10] Barnes, M.R., "Form-finding and Analysis of Prestressed Nets and Membranes”, Computers \& Structures, 1988, Vol. 30, No. 3, pp. 685-695.

[11] Wakefield, D.S., "Engineering Analysis of Tension Structures: Theory and Practice”, Engineering Structures, 1999, Vol. 21, pp. 680-690.

[12] Gosling, P.D. and Lewis, W.J., "Optimal Structural Membranes-II. Form-finding of prestressed Membranes using a Curved Quadrileteral Finite Element for Surface Definition”, Computers \& Structures, 1996, Vol. 61, No. 5, pp. 885-895.

[13] Han, S.E. and Lee, K.S., "A Study of the Stabilizing Process of Unstable Structures by Dynamic Relaxation Method”, Computers \& Structures, 2003, Vol. 81, pp. 1677-1688.

[14] Lewis, W.J., Tension Structures: Form and Behaviour, Thomas Telford, 2003.

[15] Topping, B.H.V. and Iványi, P., Computer Aided Design of Cable-membrane Structures”, Saxe-Coburg Publications, 2007

[16] Linhard, J., D'Anza, G., Bletzinger, K.-U., "Rhino-Membrane - Modern State of the Art Application for Tensile Structure Form Finding”, TensiNews, 2008, Vol. 15. 
[17] D'Anza, G., ”Rhino-Membrane in Real Modelling Problems”, Structural Membranes 2009. 2009.

[18] Maurin, B. and Motro, R., "The Surface Stress Density Method as a Form-finding Tool for Tensile Membrane”, Engineering Structures, 1999, Vol. 20, pp. 712-719.

[19] Pauletti, R.M.O. and Pimenta, P.M., "The Natural Force Density Method for the Shape Finding of Taut Structures”, Comput. Methods Appl. Mech. Engrg., 2008, Vol. 197, pp. 4419-4428.

[20] Argyris, J.H., Dunne, P.C., Angelopoulos. T. and Bichat, B., "Large Natural Strains and Some Special Difficulties due to Non-linearity and Incompressibility in Finite Elements“, Comput. Methods Appl. Mech. Engrg., 1974, Vol. 4, No. 2, pp. 219-278.

[21] Pauletti, R.M.O., Guirardi, D.M. and Deifeld, T.E.C., “Argyris's Natural Membrane Finite Element Revisited”, International Conference on Textile Composites and Inflatable Structures, Structural Membranes, 2005. 